\title{
SCHISTOSOMA MANSONI CHEZ LE MALGACHE
}

\author{
Par J. RAYNAL
}

\section{Sa fréquence. Essai de répartition géographique}

\section{de l'endémie bilharzienne à Madagascar}

La parasitose due à Schistosoma mansoni, appelée plus communément bilharziose intestinale et signalée pour la première fois à Madagascar par Girard en 1918 est, ainsi que cet auteur l'avait déjà pressenti en 1920 , une maladie endémique dans cette colonie. Cette affirmation, avec toutes les importantes séquelles qu'elle entraîne au point de vue dépistage, traitement et prophylaxie est assez peu connue, semble-t-il, ou tout au moins, n'a donné lieu qu'à des recherches très fragmentaires en vue de sa confirmation.

Girard trouve la cause bilharzienne de leur dysenterie chez trois (1918), puis chez 23 malades (1920) dont il donne la province d'origine. Hasle, tout récemment (1928) indique cette même origine bilharzienne chez 25 dysentériques traités à Ambositra.

En 1921, Morin examinant systématiquement 394 sujets présumés sains de la région de Farafangana trouve 51 porteurs de Schistosoma mansoni, soit une proportion de 13 0/0.

L'étude du parasitisme intestinal chez les troupes indigènes a incité de nombreux auteurs à rechercher parallèlement le pourcentage des porteurs de Schistosoma mansoni : MM. Léger et Pringault trouvent à Marseille, en 1921, sur 150 Malgaches, 14 porteurs de bilharzies, soit $90 / 0$. Emily, dans une imposante statistique portant sur 1.206 tirailleurs malgaches, donne 210 porteurs de Schistosoma mansoni, soit $17,410 / 0$ (1). Nous avons enfin signalé nous-même, chez les tirailleurs malgaches hospitalisés à Marseille en 1927, une proportion de $100 / 0$ de porteurs de ce parasite (14 parasités sur 139 examinés).

Ces données éparses ne permettent de se faire qu'une idée très

(1) Malheureusement, l'origine de ces 210 bilharziens n'est que très vaguement mentionnée : 75 sur 425 examinés pour la côte est, 112 sur 501 pour la côte sud, 19 sur 203 pour les Hauts-Plateaux, 4 sur 77 pour la côte ouest.

Anvales de Parasitologie, T. VII, $\mathrm{N}^{\circ} 1$ - $1^{\text {er }}$ janvier 1929 , p. 10-28. 
approximative de la répartition géographique de l'affection dans la grande île : elle peut être encore résumée à l'heure actuelle en ces quelques lignes écrites par G. Reynaud et M. Léger dans un rapport présenté au Congrès de la Santé Publique et de la Prévoyance Sociale de Marseille en 1922: "Schistosomum mansoni est fréquent dans la partie méridionale et orientale de l'île tandis qu'il parait manquer totalement dans la partie septentrionale et occidentale. »

A lire d'ailleurs les différentes publications parues, on y relève de l'une à l'autre des divergences et même des contradictions qui rendent impossible une systématisation plus nette. Si l'accord se fait sur l'infestation des provinces de Farafangana, d'Ambositra et de Fianarantsoa (Girard, Hesle, Morin, Léger et Pringault), Morin, concluant nettement à l'infestation de la région côtière orientale de Mananjary au nord, à Fort-Dauphin au sud, ajoute cependant que les Hauts-Plateaux et la zone montagneuse et boisée en demi-altitude seraient, d'après lui, indemnes (1).

MM. Léger et Pringault sont tentés de déclarer indemnes les provinces de Tananarive, Moramanga, Vatomandry et Antsirabé ; des malades de Girard (1920) proviennent pourtant de ces provinces et nous avons trouvé des porteurs de Schistosoma mansoni originaires de Vatomandry et d'Antsirabé. Dans de nombreux travaux (Girard, Morin, Raynal), la province de Fort-Dauphin est donnée comme infestée; or il est curieux de constater que Sice, dans une note récente, étudiant les principales causes de dysenterie chez les Antandroy ne parle pas de schistosomes au cours de 600 examens pratiqués (2).

La proposition même de Reynaud et Léger ne serait plus exacte en ce qui concerne la région occidentale de Madagascar : le cas de Girard en provenance d'Analalava (1920), les porteurs sains signalés par Emily (4 sur 77), notre cas de la province de Morondava semblent l'infirmer.

Dans le nord de l'île, l'absence de bilharziose est unanimement signalée dans les études qui en font mention (Emily, Raynal,

(1) Par cette formule, Morin n'a certainement en vue que les régions en altitude de la province de Farafangana. Même ainsi rectifiée, il nous semble difficile d'accepter sa thèse : nous verrons plus loin que la bilharziose semble au contraire descendre les pentes et les hautes vallées du plateau sud, et qu'en particulier la région montagneuse de Midongy du Sud est infestéc dans la proportion de plus de $900 / 0$.

(2) Ce travail de Sice, qui ne porte que sur les indigènes du pays Antandroy; " pays de sable compris entre les derniers contreforts de l'Emyrne et la mer » vient encore à l'appui de notre thèse : dans le sud de Madagascar les régions de côtes sablonnieuses sont relativement indemnes alors que les parties montagueuses présentent parfois un gros degré d'endémie. 
Reynaud et Léger). Girard a bien trouvé ses premiers cas à DiégoSuarez, mais sur des sujets provenant des provinces du Sud (1918 et 1920 ).

Pour essayer de jauger de façon plus nette l'importance que pouvait peut-être revêtir à Madagascar l'endémie bilharzienne, nous avons examiné spécialement à ce sujet, ces mois derniers, les contingents malgaches en garnison à Marseille : C. O. A. C., $15^{\circ} \mathrm{C}$. O. A. et $15^{\circ} \mathrm{S}$. I. M. (1). Nous avons examiné, pour chaque tirailleur, quatre préparations de selles non diluées entre lame et lamelle, chacune explorée entièrement au chariot mobile. Nous ne nous dissimulons pas que cette pratique est insuffisante pour dépister tous les porteurs d'œufs de Schistosoma mansoni; à défaut d'enrichissement des selles, il est classique de demander au minimum la lecture de 10 ou 12 préparations. Mais, si de ce fait notre proportion globale de parasités se trouve en-dessous de la réalité, nous ne pensons pas, la même règle ayant été suivie pour tous, que nos conclusions aient pu en souffrir.

620 tirailleurs malgaches ont été examinés : 157 ont été reconnus porteurs d'œufs de Schistosoma mansoni ; leur proportion est donc légèrement supérieure à $250 / 0$.

Malgré la sous-estimation inévitable dont nous venons de donner la raison, cette proportion est beaucoup plus forte que toutes celles données jusqu'ici, soit que le nombre d'examens, pour chaque selle, ait été plus grand, soit peut-être à cause d'une recrudescence actuelle de la bilharziose dans la grande île ; quant à la pensée d'une coïncidence qui eut pu nous mettre en présence de contingents uniquement recrutés dans les régions les plus infestées de Madagascar, elle ne résiste pas à la comparaison des différents chiffres recueillis par province, chiffres relatifs aux examens d'une part, et à la densité bilharzienne de l'autre.

Tous renseignements d'âge, de pays d'origine, de lieu de recrutement, ont été notés pour chaque tirailleur ; compte a été tenu également des migrations ayant pu survenir dans leur existence avant le service militaire. Les six tableaux suivants résument nos recherches: nous avons pensé qu'il serait intéressant de mentionner le village et le canton d'origine des malgaches parasités par Schistosoma mansoni, ces données pouvant faciliter ultérieurement sur place toutes recherches, soit de gîtes de planorbes, soit d'indigènes parasités (2).

(1) Section de commis et ouvriers d'administration coloniaux ; $15^{\mathrm{e}}$ section de commis et ouvriers militaires d'administration ; $15^{\circ}$ section d'infirmiers militaires.

(2) Nous avons conservé dans cette étude l'ancienne division administrative par province qui existait avant 1926 à Madagascar et avec laquelle nous sommes plus familiarisé. 


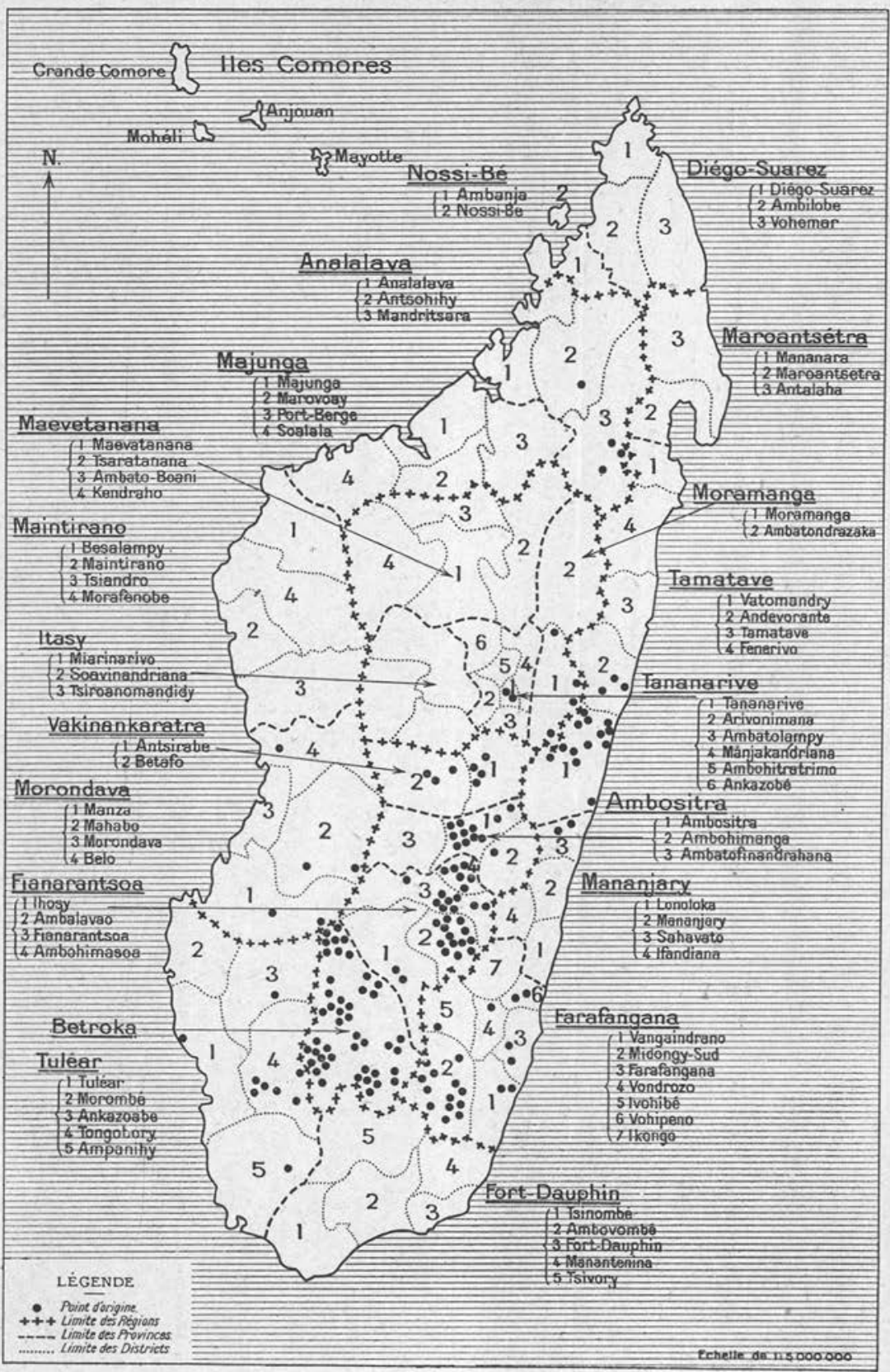




\section{RÉGION SUD DE MADAGASCAR}

Examinés : 37 (ex.). Bilharziens : 9 (Bz.). Indice : 24,32 0/0

\begin{tabular}{|c|c|c|c|}
\hline Provinces & Districts & Cantons & VII.LAgES \\
\hline $\begin{array}{c}\text { Tuléar : } \\
\text { ex., } 27-B \%, 9\end{array}$ & $\begin{array}{l}\text { Tuléar : ex,. } 10-\text { Bz., } 1 . \\
\text { Morombé : ex., } 3-\mathrm{Bz} . \text {, } \\
\text { néant. } \\
\text { Ankazoabé : ex., } 1-\text { Bz., } 1 \text {. } \\
\text { Tongobory: ex., } 11-B z ., 6 . \\
\text { Ampanihy : ex., } 2-\text { Bz., } 1 \text {. }\end{array}$ & $\begin{array}{l}\text { Manombé. } \\
\text { Andranolava. } \\
\text { Tongobory. } \\
\text { Vélotora. } \\
\text { Soamanonga. } \\
\text { Ambohimavelo. } \\
\text { Ampanihy. }\end{array}$ & $\begin{array}{l}\text { Ankodobariky. } \\
\text { Andranolava. } \\
\text { Lokahita, Anovoay. } \\
\text { Andranalava. } \\
\text { Renambé. } \\
\text { Faretsy. } \\
\text { Nahalotsy. } \\
\text { Ampilietra. }\end{array}$ \\
\hline $\begin{array}{c}\text { Fort-Dauphin : } \\
\text { ex., } 10-B z ., \\
\text { néant. }\end{array}$ & $\begin{array}{l}\text { Tsiombé : ex., } 1-\text { Bz., } \\
\text { néant. } \\
\text { Ambovombé : ex., } 1- \\
\text { Bz., néant. } \\
\text { Fort-Dauphin : ex., } 5- \\
\text { Bz., néant. } \\
\text { Manantén:na : ex., } 3- \\
\text { Bz, néant. }\end{array}$ & & \\
\hline
\end{tabular}

II. PLATEAUX SUD DE MADAGASCAR

Examinés : 170. Bilharziens : 94 . Indice : 55,30 o/o

\begin{tabular}{|c|c|c|}
\hline Provinces & Cantons & Villages \\
\hline $\begin{array}{l}\text { District autonome de Betroka : } \\
\qquad \text { ex., } 47-\text { Bz., } 45\end{array}$ & $\begin{array}{l}\text { Betroka. } \\
\text { Imahabo. } \\
\text { Isoanala. } \\
\text { Hazofotsy. } \\
\text { Sakamahily. } \\
\text { Vohiboro. } \\
\text { Benenitra. }\end{array}$ & $\begin{array}{l}\text { Razomina, Tranoara. } \\
\text { Mandarano. } \\
\text { Manisy, Ampasimainty. } \\
\text { Analabé, Bepeho, Berotsy. } \\
\text { Ambindadé, Andriamero, } \\
\text { Ambakely. } \\
\text { Atondrosy, Antananda. } \\
\text { Ampadatolana. } \\
\text { Anzao, Saliahara. } \\
\text { Andenbé. } \\
\text { Andranomanesky, Rano- } \\
\text { hendry. } \\
\text { Bivoandelaka, Keliamba- } \\
\text { koha. } \\
\text { Vohiboro. } \\
\text { Bereketa, Sakotoko. } \\
\text { Ambohitra, Ambovato. }\end{array}$ \\
\hline
\end{tabular}


II. PLATEAU SUD DE MADAGASCAR (suile)

\begin{tabular}{|c|c|c|c|}
\hline Provinces & Districts & Cantons & VILI.AgES \\
\hline \multicolumn{2}{|c|}{$\begin{array}{l}\text { Distriet autonome de Betroka: } \\
\text { ex., } 47-\mathrm{Bz} ., 45\end{array}$} & $\begin{array}{l}\text { Iampera. } \\
\text { Ranohira. } \\
\text { Vohimalaza. } \\
\text { Menamaty. }\end{array}$ & $\begin{array}{l}\text { Manasoa, Beharidrim- } \\
\text { bono. } \\
\text { Mahovaza, Kahimaza. } \\
\text { Ampiha, } \\
\text { Auravanda, Zavolo. } \\
\text { Ambatafotsy, Manan- } \\
\text { tanda. } \\
\text { Amboloandy, Mahaby. } \\
\text { Menamaty, Belinana. } \\
\text { Matambalahy, Bekizoly. } \\
\text { Ambalambé, Bekinamby. } \\
\text { Ambatohirika. }\end{array}$ \\
\hline $\begin{array}{l}\text { Fianarantsoa : } \\
\text { ex., } 76-B \% ., 30\end{array}$ & $\begin{array}{c}\text { Ihosy : } \\
\text { ex., } 2-B z ., 2 \\
\text { Ambalavao : } \\
\text { ex., 20-Bz., } 13\end{array}$ & $\begin{array}{l}\text { Ihosy. } \\
\text { Ambalavao. } \\
\text { Maroparasy. } \\
\text { Manampy. } \\
\text { Tsahamimasy. } \\
\text { Ambohimamasina. } \\
\text { Tsaronenana. } \\
\text { Vohimanombo. } \\
\text { Hlamosina. } \\
\text { Ifandana. } \\
\text { Besoa. } \\
\text { Angodogodo. } \\
\text { Tsahamanitra. } \\
\text { Fitampito } \\
\text { Isorana. } \\
\text { Ait:ıngoa. } \\
\text { Andrainjato. } \\
\text { Talata-Ampany. } \\
\text { Adonamaromaintso. } \\
\\
\text { Ambohinamboarino. } \\
\text { Ambohimasoa. } \\
\text { Alarobia-Vohiposa. } \\
\text { Antalata. } \\
\text { Sahamadio. } \\
\text { Sahave. }\end{array}$ & $\begin{array}{l}\text { laborano, Ihosy. } \\
\text { Alasora, Ambahibo. } \\
\text { Anaramboay. } \\
\text { Amana. } \\
\text { Vohitombé. } \\
\text { Vohimarino. } \\
\text { Akrefo. } \\
\text { Dondoly. } \\
\text { Ambalapeso. } \\
\text { Ambananjouma. } \\
\text { Ambohiboro. } \\
\text { Ambohidongodo. } \\
\text { Tsahamanitra. } \\
\text { Ambalahady. } \\
\text { Asanga, Itsara. } \\
\text { Ambalavo. } \\
\text { Tahitambo. } \\
\text { Ambalamanakara. } \\
\text { Adonamaromaintso, Am- } \\
\text { balasava, Ambalami- } \\
\text { soalra. } \\
\text { Ambombiry. } \\
\text { Ambohimanalika. } \\
\text { Ambatana. } \\
\text { Antsinjila. } \\
\text { Ambilanorono. } \\
\text { Ambohitsanona. }\end{array}$ \\
\hline $\begin{array}{l}\text { Ambositra: } \\
\text { ex } 26-\text { Bz., } 12\end{array}$ & $\begin{array}{l}\text { Ambositra : } \\
\text { ex., } 16-\mathrm{Bz} ., 11\end{array}$ & $\begin{array}{l}\text { Ambositra. } \\
\text { Ambohimahazo. } \\
\text { Imerina. } \\
\text { Andina. } \\
\text { Ivato. } \\
\text { Tsarasaotra. } \\
\text { Fandriana. } \\
\text { Miaranavaratra. }\end{array}$ & $\begin{array}{l}\text { Ambohiponana. } \\
\text { Fenoarivo, Sahatsivoly. } \\
\text { Ambalamirana. } \\
\text { Ambondramasina. } \\
\text { laranarivo. } \\
\text { Iatobé. } \\
\text { Ampanihy. } \\
\text { Tsahantsotry. }\end{array}$ \\
\hline
\end{tabular}


II. PLATEAUX SUD DE MADAGASCAR (suile)

\begin{tabular}{|c|c|c|c|}
\hline Provinces & Districts & Cantons & ViLlagis \\
\hline $\begin{array}{c}\text { Ambositra : } \\
\text { ex., } 26-B z ., 12\end{array}$ & $\begin{array}{l}\text { Ambohimanga : } \\
\text { ex., } 9-\mathrm{Bz} ., 1 \\
\text { Ambatofinandra- } \\
\text { bana: } \\
\text { ex, } 1-\text { Bz., néant }\end{array}$ & $\begin{array}{l}\text { Ambatomarina. } \\
\text { Ambohimitombo. }\end{array}$ & $\begin{array}{l}\text { Ambatolahy, Anvolaly. } \\
\text { Tombohonina. }\end{array}$ \\
\hline $\begin{array}{l}\text { Vakinanka- } \\
\text { ratra: } \\
\text { ex., } 21-B z ., 7\end{array}$ & $\begin{array}{c}\text { Antsirabé : } \\
\text { ex., } 13-\text { Bz., } 4 \\
\text { Betafo: } \\
\text { ex., } 8-\text { Bz., } 3\end{array}$ & $\begin{array}{l}\text { Antsirabé. } \\
\text { Belazao. } \\
\text { Andranomanelatra. } \\
\text { Betafo. } \\
\text { Ambohimanambolo. }\end{array}$ & $\begin{array}{l}\text { Antsirabé, Fiaranandara. } \\
\text { Ankerobé. } \\
\text { Morarana. } \\
\text { Ambaniharana. } \\
\text { Androvakely, Antenina. }\end{array}$ \\
\hline
\end{tabular}

\section{RÉGION EST DE MADAGASCAR}

Examinés : 150. Bilharziens : 39 . Indice : $260 / 0$

\begin{tabular}{|c|c|c|c|}
\hline Provinces & Distnicts & CantoNs & Vintages \\
\hline $\begin{array}{l}\text { Farafangana : } \\
\text { ex., } 41-\mathrm{Bz} ., 21\end{array}$ & $\begin{array}{l}\text { Farafangana : } \\
\text { ex, } 9-\text { Bz., } 2 \\
\text { Vondrozo: } \\
\text { ex., } 2-\text { Bz., } 1 \\
\text { Ivohibé: } \\
\text { ex., } 2-\text { Bz., } 1 \\
\text { Vohipeno: } \\
\text { ex., } 7-\text { Bz., } 2\end{array}$ & $\begin{array}{l}\text { Vangaindrano. } \\
\text { Vikaronga. } \\
\text { Midongy. } \\
\text { Befotaka. } \\
\text { Andranolahy. } \\
\text { Berehena. } \\
\text { Antanandava. } \\
\text { Lavoraty. } \\
\text { Itomampy. } \\
\text { Sourano. } \\
\text { Ankarana. } \\
\text { Etrotroka. } \\
\text { Karianga. } \\
\text { Ranotsara-Nord. } \\
\text { Ilakatra. } \\
\text { Fehifora. }\end{array}$ & $\begin{array}{l}\text { Vehilaba. } \\
\text { Vitjinky. } \\
\text { Midongy, Feliandro. } \\
\text { Bemena, Morafeno, Beki- } \\
\text { longo, Anvingona, Ato- } \\
\text { hajano. } \\
\text { Mahatsinjo. } \\
\text { Berehena. } \\
\text { Iberao. } \\
\text { Tramihiry. } \\
\text { Bekinana. } \\
\text { Sourano. } \\
\text { Marovaty. } \\
\text { Ambotilo. } \\
\text { Sosoba. } \\
\text { Anorosy. } \\
\text { Vohona. } \\
\text { Fehifora. }\end{array}$ \\
\hline $\begin{array}{l}\text { Mananjary : } \\
\text { ex., } 11-B \% ., 2\end{array}$ & $\begin{array}{l}\text { Lonoloka : } \\
\text { ex., } 3-\text { Bz., néant } \\
\text { Mananjary : } \\
\text { ex., } 5-\text { Bz, néant } \\
\text { Sahavato : } \\
\text { ex. } 3-\text { Bł., } 2\end{array}$ & $\begin{array}{l}\text { Sòvina. } \\
\text { Ambodilafa. }\end{array}$ & $\begin{array}{l}\text { Ilakomandroro. } \\
\text { Ambodilafa. }\end{array}$ \\
\hline
\end{tabular}




\section{RÉGION EST DE MADAGASCAR (suile)}

\begin{tabular}{|c|c|c|c|}
\hline Provinces & Districts & Cantons & Villages \\
\hline $\begin{array}{c}\text { Tamatave : } \\
\text { ex., } 62-B z ., 16\end{array}$ & $\begin{array}{c}\text { Andevorante: } \\
\text { ex., } 10-\text { Bz., } 3 \\
\\
\text { Tumatava : } \\
\text { ex., } 11-\text { Bz., néant } \\
\text { Fenerivo : } \\
\text { ex , } 19-\text { Bz., néant }\end{array}$ & $\begin{array}{l}\text { Vatomandry. } \\
\text { Antanambao. } \\
\text { Ankazofitsantrata. } \\
\text { Lohovanona. } \\
\text { Ampasimazava. } \\
\text { Tsivangana. } \\
\text { Andonabé. } \\
\text { Mas meloka. } \\
\text { Tsaravinany. } \\
\text { Ifasina. } \\
\text { Maintinandry. } \\
\text { Ambinidilana. } \\
\text { Bedara. } \\
\text { Vohibohazo. } \\
\text { Anivorano. }\end{array}$ & $\begin{array}{l}\text { Ambotofota. } \\
\text { Ankoraka. } \\
\text { Fisakalana. } \\
\text { Sahafangana. } \\
\text { Ambohimanarivo. } \\
\text { Ambihanizao. } \\
\text { Ambatomalady. } \\
\text { Fitsialana. } \\
\text { Atseranambato. } \\
\text { Ambalavontaka. } \\
\text { Ambodivohangy. } \\
\text { Ambodibokona. } \\
\text { Ambinidilana. } \\
\text { Bedara. } \\
\text { Beforo. } \\
\text { Tanambao. }\end{array}$ \\
\hline $\begin{array}{l}\text { Maroantsetra : } \\
\text { ex.,36-Bz., néant }\end{array}$ & $\begin{array}{l}\text { Mananara : } \\
\text { ex., } 17-\mathrm{Bz} \text {, néant } \\
\text { Maroantsatra : } \\
\text { ex., } 18-\mathrm{B} z ., \text { néant } \\
\text { Antalaha : } \\
\text { ex., } 1-\text { Bz., néant }\end{array}$ & & \\
\hline
\end{tabular}

IV. HAUTS-PLATEAUX, CENTRE ET NORD

Examinés : 192. Bilharziens : 6 . Indice : 3,12 o/o

\begin{tabular}{|c|c|c|c|}
\hline Provinces & Districts & CANToNs & Villatges \\
\hline $\begin{array}{l}\text { Moramanga: } \\
\text { ex., } 58-\text { bz, } 4\end{array}$ & $\begin{array}{c}\text { Moramanga : } \\
\text { ex., } 34-B z ., 4 \\
\text { Ambatondrazaka: } \\
\text { ex., } 24-\text { Bz., néant }\end{array}$ & $\begin{array}{l}\text { Andanobé-Nord. } \\
\text { Ampasimbé. } \\
\text { Ampasimazava. } \\
\text { Andaingo. }\end{array}$ & $\begin{array}{l}\text { Ambodivatoanana. } \\
\text { Sanantinga } \\
\text { Benavona. } \\
\text { Ambohimanarivo. }\end{array}$ \\
\hline $\begin{array}{l}\text { Tananarive: } \\
\text { ex., } 97-\mathrm{Bz} ., 2\end{array}$ & $\begin{array}{c}\text { Tananarive : } \\
\text { ex., } 51-\mathrm{Bz} ., 2 \\
\text { Arivonimana : } \\
\text { ex , } 6-\mathrm{Bz} ., \text { néant } \\
\text { Ambotolampy : } \\
\text { ex., } 10-\mathrm{Bz} \text {., néant }\end{array}$ & Tananarive. & Tananarive, Isotra. \\
\hline
\end{tabular}

Annales de Parasitologie, $\mathrm{T}$. VII, $\mathrm{x}^{\circ} 1 .-1^{\mathrm{er}}$ janvier 1929. 
IV. HAUTS-PLATEAUX, CENTRE ET NORD (suile)

\begin{tabular}{|c|c|c|c|}
\hline Provinces & Distnicts & Cantons & VILI.AgES \\
\hline $\begin{array}{l}\text { Tananarive : } \\
\text { ex., } 97-\text { Bz., } 2\end{array}$ & $\begin{array}{l}\text { Manjakandriana : } \\
\text { ex., } 16-\text { Bz., néant } \\
\text { Ambohitratrimo : } \\
\text { ex., } 11-\text { Bz., néant } \\
\text { Ankazobé : } \\
\text { ex., } 3-\text { Bz., néant }\end{array}$ & & \\
\hline $\begin{array}{c}\text { Itasy : } \\
\text { ex., } 18-\text { Bz.., néant }\end{array}$ & $\begin{array}{l}\text { Miarinarivo : } \\
\text { ex., } 2-\text { Bz., néant } \\
\text { Soanivandriano : } \\
\text { ex., } 15-\text { Bz., néant } \\
\text { Tsiroanomandidy : } \\
\text { ex., } 1-\text { Bz., néant }\end{array}$ & & \\
\hline $\begin{array}{l}\text { Maevatanama: } \\
\text { ex., } 19-\text { Bz., néant }\end{array}$ & $\begin{array}{c}\text { Maevatanana : } \\
\text { ex., } 5-\text { Bz., nèant } \\
\text { Tsaratanana : } \\
\text { ex., } 2-\text { Bz., néant } \\
\text { Amboto-Boani : } \\
\text { ex., } 12-\text { Bz., nèant }\end{array}$ & & $=$ \\
\hline
\end{tabular}

V. CÔTE OUEST DE MADAGASCAR

Examinés : 44. Bilharziens : 9. Indice : $20,500 / 0$

\begin{tabular}{|c|c|c|c|}
\hline Provinces & Districts & Cantons & 1 Villlages \\
\hline $\begin{array}{l}\text { Morondava : } \\
\text { cx., } 7-\text { Bz., } 5\end{array}$ & $\begin{array}{c}\text { Manza : ex., } 3-\text { Bz., } 2 \\
\text { Mahabo : ex., } 2-\text { Bz., } 2 \\
\text { Morondava : } \\
\text { ex , } 1-\text { Bz., nèant } \\
\text { Belo : ex., } 1-\text { Bz., } 1\end{array}$ & $\begin{array}{l}\text { Manza. } \\
\text { Berorona. } \\
\text { Mandronarivo. } \\
\text { Mandabé. } \\
\text { Sérinam. }\end{array}$ & $\begin{array}{l}\text { Miari. } \\
\text { Akazoabé. } \\
\text { Mandronarivo. } \\
\text { Analamahavelo. } \\
\text { Andemba. }\end{array}$ \\
\hline $\begin{array}{l}\text { Maintirano: } \\
\text { ex., } 1-\text { Bz., néant }\end{array}$ & $\begin{array}{c}\text { Besalampy : } \\
\text { ex., } 1-\text { Bz., néant }\end{array}$ & & \\
\hline $\begin{array}{c}\text { Majunga : } \\
\text { ex.,14-Bz.,néant }\end{array}$ & $\begin{array}{l}\text { Majunga : } \\
\text { ex., } 2-\text { Bz., néant } \\
\text { Marovoay : } \\
\text { ex., } 5-\text { Bz., néant } \\
\text { Port-Bergé : } \\
\text { ex., } 7-\text { Bz., néant }\end{array}$ & & \\
\hline $\begin{array}{l}\text { Analalava: } \\
\text { ex., } 22-B z ., 4\end{array}$ & $\begin{array}{c}\text { Analalava : } \\
\text { ex., } 3-\text { Bz., néant } \\
\text { Antsolihy : } \\
\text { ex., } 7-\text { Bz., } 1 \\
\text { Mandritsara : } \\
\text { ex., } 12-\mathrm{Bz} ., 3\end{array}$ & $\begin{array}{l}\text { Befandriana. } \\
\text { Mandritsara. } \\
\text { Marotandro. } \\
\text { Antsirabé. }\end{array}$ & $\begin{array}{l}\text { Soamango. } \\
\text { Andramanahady. } \\
\text { Amialavary. } \\
\text { Tataka. }\end{array}$ \\
\hline
\end{tabular}


VI. RÉGION NORD ET COMORES

Examinés : 27. Bilharziens : néant. Indice : néant

\begin{tabular}{|c|c|c|c|}
\hline Provinces & Districts & Cantoxs & ViLt.age: \\
\hline $\begin{array}{c}\text { Nossi-Bé : } \\
\text { ex., 4-Bz., néant }\end{array}$ & $\begin{array}{c}\text { Ambanja : } \\
\text { ex., } 4-B z ., \text { néant }\end{array}$ & & \\
\hline $\begin{array}{l}\text { Diego-Suarez: } \\
\text { ex.,14 - Bz., néant }\end{array}$ & $\begin{array}{c}\text { Diego-Suarez : } \\
\text { ex., } 2-\text { Bz., néant } \\
\text { Ambilobé : } \\
\text { ex., } 5-\text { Bz., néant } \\
\text { Vohémar : } \\
\text { ex., } 7-\text { Bz., néant }\end{array}$ & & \\
\hline $\begin{array}{l}\text { Comores (iles) } \\
\text { ex.,9-B\%, néant }\end{array}$ & $\begin{array}{l}\text { Mayolte : } \\
\text { ex., } 1-\text { Bz., néant } \\
\text { Grande Comore : } \\
\text { ex., } 8-\mathrm{Bz} \text {., néant }\end{array}$ & & \\
\hline
\end{tabular}

Ainsi, nous avons trouvé douze provinces atteintes dans leur contingent par Schistosomà mansoni: Tuléar dans le Sud ; Betroka, Fianarantsoa, Ambositra, Vakinankaratra, Tananarive et Moramanga sur les Hauts-Plateaux ; Farafangana, Mananjary et Tamatave sur la côte est ; Morodava et Analalava sur la côte ouest.

Neuf provinces ont été reconnues indemnes: Fort-Dauphin, Maroantsetra, Itasy, Maevatanana, Maintirano, Majunga, Nossi-Bé, Diégo-Suarez et les Comores ; mais pour certaines d'entr'elles le nombre des tirailleurs examinés n'est pas suffisant pour pouvoir conclure fermement à la non-endémie.

Une topographie régionale nous permettra de mieux situer les principaux foyers de l'affection :

C'est dans la partie toute méridionale du plateau central de Madagascar que se trouve la région la plus fortement infestée; le véritable noyau de la bilharziose est là, dans les hautes vallées du pays Baria, de l'Antsansa et du Barabé qui remontent vers les massifs du Tsiombivositra, de l'Ivakoany et du Horombé : cette région correspond administrativement aux trois districts de Betroka, d'Ihosy et de Midongy du Sud qui donnent dans leur ensemble des chiffres éloquents: 60 bilharziens sur 63 examinés, soit un pourcentage de $950 / 0$.

De ce foyer, suivant les pentes et les vallées, l'endémie déborde vers les régions de Morondava et de Tuléar à l'ouest, de Farafan- 


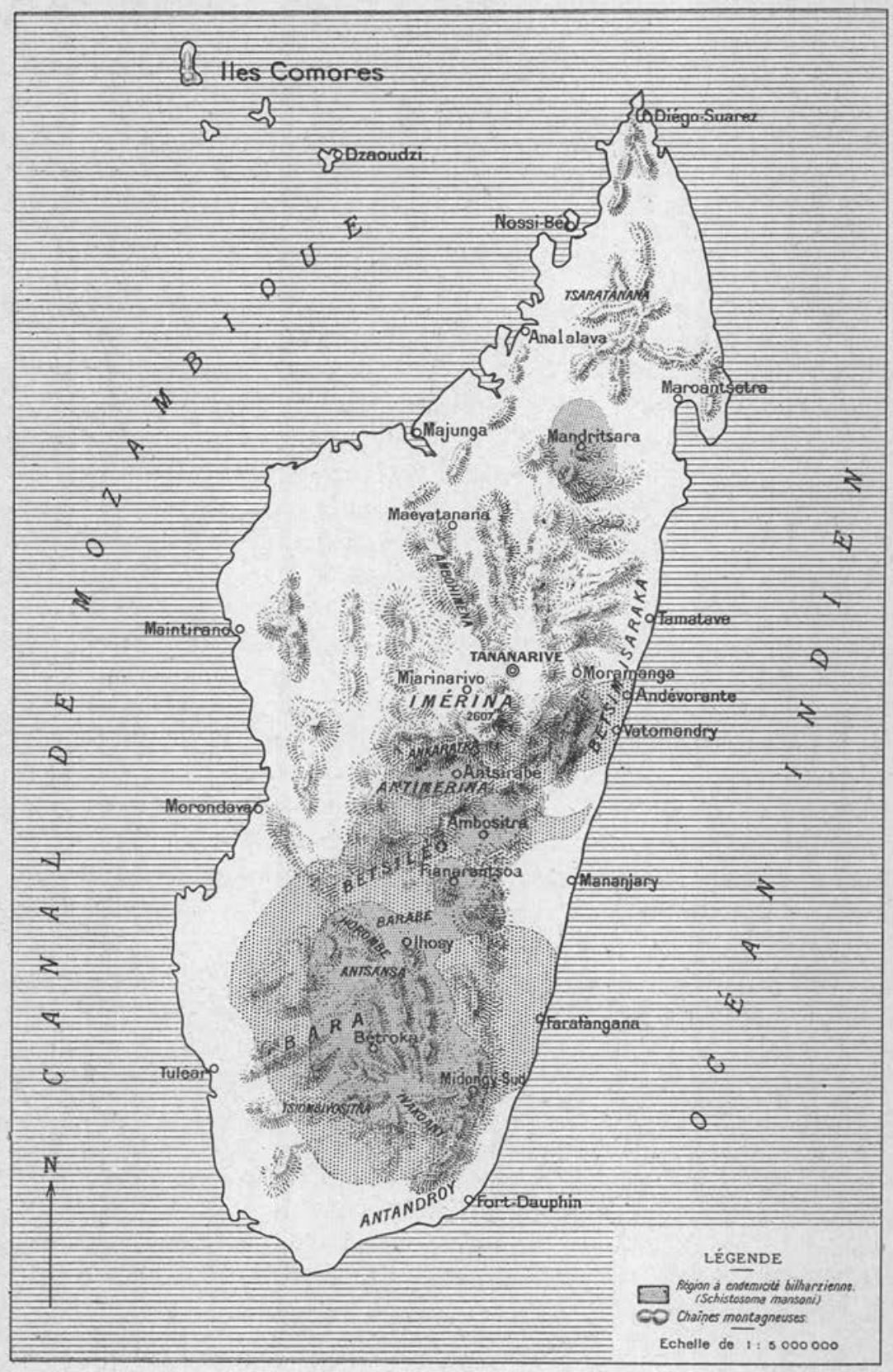


gana à l'est et de Fort-Dauphin au sud, mais ne frappe presque exclusivement là que les contreforts montagneux; les régions sablonneuses du sud en sont indemnes. C'est pour cette raison que Sice ne parle pas de bilharziose intestinale à Fort-Dauphin et que nos résultats sont négatifs pour cette province: aucun de nos tirailleurs ne provenait du district de Tsivory, situé dans la partie la plus montagneuse; au contraire, dans notre précédente étude, nous avions trouvé trois bilharziens ayant cette origine.

Au nord de cette vaste région d'altitude où la proportion des porteurs de Schistosoma mansoni est si massive, l'endémie se continue, moins dense, sur le plateau central, alimentée par deux foyers principaux qui se trouvent au cœur du pays Betsiléo: le foyer Ambalavao-Fianarantsoa et le foyer d'Ambositra, ce dernier poussant un prolongement vers la côte est, à la limite des provinces de Mananjary et de Tamatave.

Plus haut, dans l'Antimerina, existe un quatrième foyer moins important que les précédents : Antsirabé-Betafo. Ce foyer déborde largement sur la côte est, vers Vatomandry et Andévorante, en écornant la partie toute méridionale de la province de Moramanga.

Au nord du massif de l'Ankaratra, la bilharziose doit être localisée en des point's très limités ; nous n'y pouvons signaler que Tananarive même ( 2 cas sur 51 examinés), mais du côté des sources de la Betsiboka, dans la région comprise entre Fihaonana et Vohilena, le $\mathrm{D}^{\mathrm{r}}$ Juneau nous entretenait récemment d'une maladie connue localement sous le nom de "fièvre du voini-zongo », dans laquelle il y aurait tout lieu de penser que Schistosoma mansoni fut en cause : les malades, certains de 20 à 30 ans, présentent une sorte de syndrome de Banti, cirrhose hépatique avec splénomégalie et ascite fréquente.

Enfin il y aurait, dans les régions d'altitude de la province d'Analalava, point qui avait déjà donné des cas de bilharziose intestinale à Girard, un dernier foyer, celui de Befandriana-Mandritsara où l'affection paraîtrait aussi assez localisée.

Cette esquisse géographique de la bilharziose intestinale à Madagascar ne correspond qu'à une vue d'ensemble très générale; dans l'étude épidémiologique de cette affection, les moindres détails ont leur importance, et, du fait de l'habitat de l'hôte intermédiaire, on trouvera, par l'enquête menée sur place, des points d'infestation très limités, voisins ou au contraire éloignés les uns des autres dans les régions que nous donnons plus haut comme atteintes.

C'est donc sur place et par des recherches concomitantes portant aussi bien sur les selles des habitants d'une région que sur la faune 
malacologique des ruisseaux ou des petites mares, avec expérimentation au laboratoire, qu'on arrivera à fixer les points exacts de contamination, les foyers locaux de bilharziose et ceux où l'infestation risque de gagner du terrain. Ces recherches auraient le mérite de ne point rester stériles car, en connaissance de cause, des mesures prophylactiques tendant à restreindre l'endémie pourraient être envisagées. Elles seraient nécessaires aussi pour fixer plus étroitement et compléter la géographie médicale de l'affection à Madagascar, telle que nous l'avons définie.

Celle-ci ne saurait même pas avoir la prétention d'être définitive : ce n'est pas que nous pensions, malgré les conditions défavorables dans lesquelles nous étions placé, qu'elle soit passible de grosses causes d'erreur (1), mais nous ne doutons pas que l'endémie bilharzienne soit actuellement en progression à Madagascar : nous n'en voulons pour preuves, que, comparés aux résultats antérieurs, la densité des foyers que nous avons signalés et le pourcentage plus grand de parasités dépistés dans un lot de tirailleurs déjà sélectionnés à leur départ.

Apportant cette notion qu'un quart de ces indigènes étaient porteurs de Schistosoma mansoni, il nous a paru d'un certain intérêt d'attirer l'attention sur la topographie générale de l'endémie bilharzienne à Madagascar, telle qu'elle se constituait sous nos yeux du fait de nos recherches, sur l'existence de quelques foyers indiscutables de bilharziose, en particulier du foyer sud de la région Betroka-Midongy-Thosy, très important et non encore signalé, enfin sur l'utilité que présenterait une étude plus approfondie des causes épidémiologiques faite sur place, en raison de la fréquence avec laquelle Schistosoma mansoni parasite le Malgache.

\section{Considérations cliniques}

L'examen microscopique des selles nous ayant permis de dépister un grand nombre de porteurs de Schistosoma mansoni chez les tirailleurs malgaches en service à Marseille, nous nous sommes demandé dans quelle mesure cette infestation des vaisseaux portes dans laquelle le mode d'élimination des œufs joue le principal rôle,

(1) Par les nombreux renseignements recueillis et par la différence du taux des parasités suivant telle ou telle origine, il est impossible, par exemple, qu'il y ait eu contamination dans un lieu de passage commun ou dans un lieu de rassemblement des recrues avant ou au moment de leur départ pour Marseille. Ces lieux de rassemblement d'ailleurs, Tananarive, Tamatave, Diégo-Suarez et Majunga, sauf en ce qui concerne le premier, n'appartiennent pas à la zone endémique. 
pouvait être supportée par eux. Existait-il chez eux des signes cliniquement perceptibles qui puissent créer un véritable état morbide, soit du côté de l'intestin, soit du côté de la vessie, de la rate ou du foie ?

Il ne faut pas perdre de vue que nous avons effectué ces recherches chez des sujets jeunes, pour la plupart bien constitués, déjà sélectionnés par deux examens médicaux, dont le dernier, en période d'incorporation, doit être particulièrement sévère. Cette étude laisse par conséquent de côté les éléments les plus intéressants, les jeunes gens éliminés, soit pour mauvais état général, soit peut-être pour splénomégalie ou cirrhose hépatique. De même que pour les recherches épidémiologiques, l'avantage de pouvoir reprendre cette étude sur place offrirait un gros intérêt.

De plus, les tirailleurs examinés appartiennent à des classes de recrutement différentes, s'échelonnant de 1909 à 1928 : un grand nombre ayant déjà passé 2 et 3 ans en France, d'autres ayant séjourné dans la métropole à plusieurs reprises par périodes de 3 ans, nous devions nous demander aussi si cette parasitose se comportait comme nous pensons que se comportent chez les indigènes en général la plupart des parasitoses intestinales. Le séjour hors des pays d'endémicité arrive-t-il à stériliser ou tout au moins à restreindre d'une façon appréciable le nombre des parasités et celui des parasites hébergés ?

1) Etat intestinal. - Le Malgache, insouciant, ne s'observe guère quand les symptômes sont peu accusés. De plus, en pays Bara notamment, où se recrutent de nombreux porteurs de Schistosoma mansoni, l'indigène est très endurant au mal. Aussi les antécédents avoués de nos bilharziens sont-ils peu chargés : 25 à $300 / 0$ seulement accusent dans leur enfance ou leur adolescence des crises dysentériques peu sévères ayant cessé d'elles-mêmes très vite. Leur parasitose a donc été, dans la plupart des cas, bien tolérée ou du moins leur a-t-elle passé complètement inaperçue.

Nous n'avons pas pratiqué de rectoscopie.

L'examen macroscopique des selles où nous avons recherché et trouvé l'œuf du parasite peut-il donner des renseignements ? Très difficilement, car $640 / 0$ de nos bilharziens sont en outre porteurs de vers intestinaux dont le nombre et la diversité varient. C'est ainsi que nous avons trouvé dans nos recherches : 


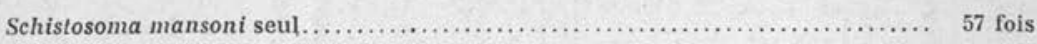

Schistosoma mansoni et uncinaires (Ankylostomum ou Necator) ............ 29 fois Sch. mansoni et trichocéphales.................................... 33 fois

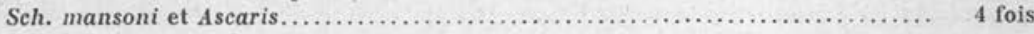

Sch. mansoni, uncinaires et trichocéphales...................... 25 fois

Sch. mansoni, Ascaris et trichocèphales ............................ 2 fois

Sch. mansoni, uncinaires et Ascaris................................ 1 fois

Sch. mansoni, uncinaires, Ascaris et trichocéphales..................... 5 fois

Sch. mansoni, uncinaires, trichocéphales et anguillules ................. 1 fois

Aussi, dans les cas de parasitoses associées est-il très difficile de définir exactement la part qui revient aux schistosomes ou aux autres parasites intestinaux, quand on se trouve en présence de selles dysentériformes, glaireuses ou mucoso-sanglantes.

Ces dernières, d'ailleurs, ont été l'exception au cours de nos recherches. Nous avons les résultats macroscopiques de 118 selles bilharziennes; elles peuvent se répartir ainsi :

a) selles normales, soit bien moulées, soit légèrement pâteuses, mais sans trace de glaires ou de sang : 59, chez lesquelles on note une association parasitaire dans 27 cas. La fréquence des œufs de Schistosoma mansoni par préparation est habituellement minime : quatre préparations sont parfois nécessaires pour déceler un œuf ou deux, mais le plus souvent on compte un et jusqu'à 4 et 5 œufs par préparation ;

b) selles bien moulées ou légèrement pâteuses, mais enrobées de mucus le plus souvent sanguinolent : 48. Le mucus se présente, soit à l'état de traces discrètes, soit plus abondant, venant parfois recouvrir la selle d'un véritable crachat rectal et souvent d'un agglomérat de mucus et de desquamation donnant à première vue un aspect tumoral, le nombre des œufs dans ces selles oscille de 1 à 15 par préparation : ils sont rarement moins ou plus nombreux que ces chiffres. Dans 29 cas, il y a association parasitaire ;

c) selles diarrhéiques et dysentériformes : 8 dans lesquelles il y a 7 associations parasitaires : on y rencontre habituellement de nombreux œufs de Schistosoma mansoni, de 5 à 20 en moyenne par préparation ;

d) selles dysentériformes franches : 3 avec 3 associations d'helminthiases ; le nombre des bilharzies par préparation est élevé. 
L'association parasitaire semble dominer dès que la selle est macroscopiquement pathologique, mais on doit noter cependant que ces selles pathologiques contiennent habituellement le nombre le plus élevé d'œufs de Schistosoma mansoni.

$\mathrm{Au}$ contraire, les selles qui se rapprochent de la normale englobent le plus grand nombre de cas (plus de la moitié) où Schistosoma mansoni est le seul parasite trouvé : les selles sont alors bien moulées ou légèrement pâteuses, enrobées d'un mucus sanguinolent plus ou moins discret ; on retrouve d'ailleurs ces mêmes caractères dans les selles de malgaches parasités simplement par des uncinaires ou plutôt par des Ascaris ou des anguillules, mais dans ces derniers cas, il nous a semblé que les glaires contenaient plus exceptionnellement du sang.

Quoi qu'il en soit, on voit qu'il serait prématuré, en raison de l'addition parasitaire intestinale et de l'inconstance avec laquelle la bilharziose s'accompagne de selles pathologiques, de se baser sur le simple aspect macroscopique des selles pour dépister les porteurs de Schistosoma mansoni. Cette opinion d'ailleurs est classique.

La glaire mucoso-sanglante qui quelquefois enrobe discrètement la selle laissera soupçonner l'œuf à éperon latéral. Mais la certitude est seule apportée par l'examen microscopique.

Celui-ci nous a permis de faire en outre les constatations suivantes :

Très rarement, même quand les selles avaient été conservées quelques heures, nous avons pu voir le miracidium éclos à côté de coques flétries et vides : le cas s'est produit exactement deux fois et, contrairement à ce que l'on pourrait penser, ce n'était pas dans des cas de selles diarrhéiques. Cette éclosion du miracidium est au contraire fréquente chez Schistosoma hæmatobium, même dans les urines non additionnées d'eau.

D'autre part nous avons vu très souvent que dans des selles enrobées de mucus, les œufs se trouvaient surtout dans la partie fécale, tandis que la partie glaireuse en contenait bien moins ou pas du tout (1).

2) Atteinte splénique. - La rate serait habituellement hypertrophiée chez de nombreux sujets porteurs de Schistosoma mansoni en

(1) Le Professeur Brumpt, à qui nous avons signalé le fait, pense que le passage des oufs dans le tube digestif aurait lieu dans ces cas non pas au niveau des parties basses du gros intestin, mais peut-être beaucoup plus haut dans le tractus intestinal; l'expérimentation en effet de Schistosoma hrmatobium sur le hérisson laisse voir des lésions, donc passage des œufs, non seulement au niveau de l'estomac et de l'intestin terminal de l'animal, mais encore dans toute la longueur du tube digestif. Dans certains cas les œufs pourraient donc être très intimement brassés avec les selles. 
Afrique et en Amérique, et aussi chez certains animaux de laboratoire sur lesquels est expérimenté Schistosoma hæmatobium (Brumpt). Nous avons recherché par la palpation et par la percussion de l'hypochondre gauche la possibilité de cette atteinte splénique chez les tirailleurs qui nous ont paru les plus fortement parasités.

Un facteur est susceptible cependant de fausser nos résultats et il est difficile de ne pas en faire état : le paludisme sévit de façon sévère en certains points de Madagascar et certaines hypertrophies spléniques pourraient revendiquer cette origine. Aussi, pour avoir un terme de comparaison, avons-nous fait les mêmes recherches sur un lot témoin de 50 malgaches non parasités. Les résultats en ont été les suivants :

\begin{tabular}{|c|c|c|c|c|c|}
\hline \multirow{2}{*}{ Catígories } & \multirow{2}{*}{ ExamiNÉs } & \multicolumn{4}{|c|}{ RAtes } \\
\hline & & nulles & petites & moyennes & grosses \\
\hline $\begin{array}{l}\text { Non-bilharziens n'accusant } \\
\text { pas d'antécédents palustres. }\end{array}$ & 50 & 44 & 4 & 2 & \\
\hline $\begin{array}{r}\text { Bilharziens accusant paludisme. } \\
\text { n'accusant pas ... }\end{array}$ & $\begin{array}{l}45 \\
48\end{array}$ & $\begin{array}{l}32 \\
42\end{array}$ & $\begin{array}{r}10 \\
5\end{array}$ & $\begin{array}{l}3 \\
1\end{array}$ & \\
\hline Total... & 93 & 74 & 15 & 4 & \\
\hline
\end{tabular}

La proportion des hypertrophies spléniques chez les bilharziens, en tenant compte des antécédents palustres toujours sujets à caution quand ces renseignements proviennent d'indigènes, ne semble pas nous donner de grosse différence, si on la compare à celle des témoins. Si notre impression est que l'atteinte splénique des bilharziens est légèrement plus accusée, nous ne nous croyons pas autorisé à conclure fermement.

3) Etat du foie. - L'examen du foie ne nous a jamais révélé d'atteinte hépatique, notre attention ayant été spécialement attirée sur la possibilité d'une cirrhose atrophique.

4) Etat de la vessie. - Nos tirailleurs parasités par Schistosoma mansoni ont été très affirmatifs : jamais dans leurs antécédents ils ne se souviennent avoir uriné du sang.

La possibilité d'une infection vésicale chez eux n'a été jugée que par l'examen des urines; aucune cystoscopie n'a été pratiquée. 
Les urines ont été examinées, macroscopiquement et microscopiquement chez 60 porteurs de parasites :

Macroscopiquement nous avons eu : 55 urines claires, 3 urines légèrement louches, 2 urines très louches à l'émission. Aucune ne présentait d'apparence hématique.

Microscopiquement et après centrifugation : absence dans toutes d'œufs de Schistosoma et d'hématies, sédiment normal dans 54 cas, quelques leucocytes dans 4 cas, très nombreux leucocytes dans les deux cas où les urines étaient très louches ; ces deux cas concernaient des sujets atteints d'infection blennorragique, la présence de gonocoques ayant été mise en évidence sur frottis.

5) Fréquence des porteurs de Schistosoma mansoni suivant leur séjour en France :

Le tableau suivant semble démontrer que le séjour dans la métropole n'a aucune influence sur la biologie du plathelminthe qui parasite les ramifications portes des malgaches :

\begin{tabular}{|c|c|c|c|}
\hline DIFfÉrentes CatÉGories & Examinés & BHLHARZIENS & POURCENTAGE \\
\hline $\begin{array}{l}\text { Classe } 1928 \text {, venant d'arriver en France...... } \\
\text { Classe } 1927 \text {, ayant un an de séjour........... } \\
\text { Classe } 1926 \text {, ayant deux ans de séjour....... } \\
\text { Classe } 1925 \text {, ayant trois ans de séjour........ } \\
\text { Classes } 1909 \text { à } 1924 \text {, ayant accompli plusieurs } \\
\text { sèjours de } 3 \text { ans en France................ }\end{array}$ & $\begin{array}{r}135 \\
122 \\
131 \\
58\end{array}$ & $\begin{array}{l}27 \\
12 \\
60 \\
22\end{array}$ & $\begin{array}{l}20 \% \\
9,83 \% \\
45,80 \% \\
37,90 \% \\
20,68 \%\end{array}$ \\
\hline
\end{tabular}

En somme, une certaine proportion $(37,580 / 0)$ des bilharziens dépistés appartient à la catégorie des porteurs de germe sains ; la bilharziose évoluant par poussées, nous émettons des réserves sur la valeur intangible du terme " porteur de germe sain » dans ces cas.

Pour les autres, les selles sont discrètement (le plus souvent) ou intensément (très rarement) mêlées de glaires sanguinolentes, mais dans la plupart des cas il y a association avec des helminthiases intestinales.

Il n'y a pas, semble-t-il, chez nos porteurs de Schistosoma mansoni de retentissement splénique. Le foie et la vessie ne sont pas touchés.

Enfin le séjour mème prolongé en France n'intervient pas pour réduire le pourcentage des porteurs du parasite. 


\section{BibLIOGRAPHIE}

Brumpt. - Réalisation expérimentale du cycle complet de Schistosoma hremalobium. Ann. de Parasitologie, VI, 1928, p. 440.

Emily. - La parasitose intestinale chez les indigènes du Corps d'Armée Colonial. Ann. de méd. et de pharm. coloniales, XXIV, 1926, p. 370.

Girard: - Sur l'existence à Madagascar de la dysenterie bilharzienne à Schistosomum americanum ou mansoni. Bull. Soc. pathol. exotique, XI, 1918, p. 34.

- La Bilharziose américaine à Madagascar. Ann. de méd. et de pharm.-coloniales, XVIII, 1920 , p. 62.

Hasce. - La Bilharziose intestinale sur les Hauts-Plateaux à Madagascar. Bull. Soc. pathol. exotique, XXI, 1928, p. 380.

LÉger (M.) et Pringault. - Helminthiase intestinale et bilharziose à Schistosomum mansoni à Madagascar. Bull. Soc. pathol. exolique, XIV, 1921, p. 247.

Morin (G.-H.). - Sur la présence d'œufs de Schistosomum mansoni à Madagascar dans les selles d'individus sains cliniquement. - Bull. Soc. pathol. exotique, XIV, 1921, p. 229

Raynal (J.). - Schistosomum mansoni chez les malgaches, in Note sur le parasitisme par douves et bilharzies. Soc. de méd. et d'hyg. coloniales et navales de Marseille, Marseille Médical no 9, 25 mars 1928, p. 423

Reynaud (G.) et LÉger (M.). - Les Bilharzioses ou Schistosomoses dans les colonies françaises. Congrès de la Santé Publique et de la Prévoyance Sociale de Marseille, 11-17 septembre 1922, p. 25.

Sice. - Le parasitisme intestinal dans le Sud de Madagascar. Bull. Soc. pathol. exotique, XX, 1927, p. 464.

Laboraloire de Bactériologie el de Parasitologie de l'Ecole d'Application du Service de Santé des Troupes Coloniales. 